\title{
Multiple-target tracking and track management for an FMCW radar network
}

\author{
Dae-Bong Kim ${ }^{1}$ and Sun-Mog Hong ${ }^{2 *}$
}

\begin{abstract}
A multiple-target tracking problem for a frequency-modulated continuous-wave (FMCW) radar network is formulated and an integrated track management system is presented to solve the tracking problem in the presence of clutter. The FMCW radar network obtains beat frequency measurements with multiple collocated radars, each transmitting a sequence of chirps. The beat frequency measurements are associated to tracks directly in the beat frequency measurement space. The direct association eliminates range/range-rate calculations and multilateration processing, and it allows to process beat frequency measurements sequentially on a chirp by chirp basis. The sequential processing effectively decomposes the measurement-to-track association problem into a series of two-dimensional assignment problems that can be solved with much less computational effort. The solution to the measurement-to-track association problem is utilized to initiate and form new tracks and to update or delete existing tracks. Monte Carlo simulations were performed to evaluate the performance of the track management system.
\end{abstract}

Keywords: FMCW radar networks; Track management; Multiple-target tracking

\section{Introduction}

Millimeter-wave frequency-modulated continuous-wave $(\mathrm{FMCW})$ radars have a great popularity in diverse applications owing to their simplicity, low cost, and robustness in rain, humidity, fog, and dusty conditions [1,2], and they are often deployed as medium- and long-range radars for the purpose of driver and transportation safety $[3,4]$. The radars are also utilized in ground and maritime surveillance systems for tracking targets $[1,5,6]$. The surveillance systems employ a track management system to maintain individual targets under track. In this work, the track management is considered for multiple collocated FMCW radars in a networked form, which can cover a larger surveillance area and ensure a higher reliability and better performance in detection and tracking. The FMCW radar system obtains beat frequency measurements with multiple collocated radars, each transmitting a sequence of chirps, and uses the measurements to update individual tracks. In tracking multiple targets in the presence of clutter, however, it is unknown which beat frequency measurement originated from which target track and it is

\footnotetext{
*Correspondence: smhong@ee.knu.ac.kr

${ }^{2}$ School of Electronics Engineering, Kyungpook National University, 1370 Sankyuk-dong, Pook-gu, Daegu 702-701, South Korea

Full list of author information is available at the end of the article
}

required to establish correspondence between measurements and tracks for track updates.

There are three possible approaches to the measurement-to-track association. It is possible to associate tracks with measurements in the range/range-rate space, in the target state space, or in the beat frequency measurement space [7]. The measurement-to-track association in the range/range-rate space requires to determine the range/range-rate of each possible target using beat frequency measurements from chirps of a radar. In the case of a single target with no clutter, one can easily determine the range and range rate of the target using two or more beat frequency measurements from it. In tracking multiple targets, however, it is nontrivial to determine the range/range-rate for each individual target. It requires to associate beat frequency measurements that originated from a common target among measurements from different targets and different chirps, taking into account nonunity probability of target detection. This approach is prone to incorrect association that causes ghost targets $[7,8]$. The measurement-to-track association in the target state space additionally requires multilateration processing. In the beat frequency measurement space, we can associate beat frequency measurements directly to tracks and update each track with its associated

\section{黑 Springer}

(c) $2013 \mathrm{Kim}$ and Hong; licensee Springer. This is an Open Access article distributed under the terms of the Creative Commons Attribution License (http://creativecommons.org/licenses/by/2.0), which permits unrestricted use, distribution, and reproduction in any medium, provided the original work is properly cited. 
measurement. This direct association and track updates eliminate range/range-rate calculations and multilateration processing. As a consequence, the association in the beat frequency measurement space is robust and dramatically reduces the probability of ghost targets under the circumstances where the probability of detection is low and different depending on radars in the network [7].

In this paper, we present a track management system for solving the multi-target tracking problem for an FMCW radar network, based on the direct association and track updates. The beat frequency measurements are processed sequentially on a chirp by chirp basis in our track management system to initiate new tracks and update established ones. The sequential processing effectively decomposes the measurement-to-track association problem into a series of two-dimensional (2-D) assignment problems that can be solved with much less computational effort. The decomposition reduces the exponential time complexity of a higher-dimensional assignment problem into $O\left(n^{2}\right)$ in the number of measurements $n$ for most scenarios $[9,10]$. We implemented an optimal algorithm to solve the 2-D assignment problem. Our track management system utilizes the solution to initiate and form new tracks and to update or delete existing tracks based on their update records. Monte Carlo simulations were performed to evaluate the proposed track management system. To our knowledge, this problem formulation and integrated track management system for an FMCW radar network presented in this paper have not been reported in any earlier work.

The organization of this paper is as follows. In Section 2, we formulate the target tracking problem for the direct use of beat frequency measurements from an FMCW radar network and present an implementation based on the extended Kalman filter to estimate target position and velocity. The data association and track management are described in Section 3. Section 4 presents Monte Carlo simulations and results. Finally, we conclude in Section 5.

\section{Tracking with FMCW radar networks}

FMCW radars transmit a sequence of waveform signals with up and down chirps. Let $m$ and $j$ denote the indices of the $m$ th radar and $j$ th chirp, respectively. It is assumed that a target, if detected from a chirp signal, produces one beat frequency corresponding to the chirp. Figure 1 shows detections and misses of two targets along a sequence of four chirps of an FMCW radar. Let $i(j, m)$ denote the index of the $i(j, m)$ th beat frequency measurement from radar $m$ and chirp $j$, and let $t$ denote the index of the $t$ th target (or termed track interchangeably). Denote by $f_{i(j, m), j, m}$, $r_{i(j, m), j, m}$, and $\dot{r}_{i(j, m), j, m}$ the true beat frequency, range, and range rate of measurement $i(j, m)$ from radar $m$ and chirp $j$, respectively. Denote by $\Delta f_{j, m}$ the sweep frequency of

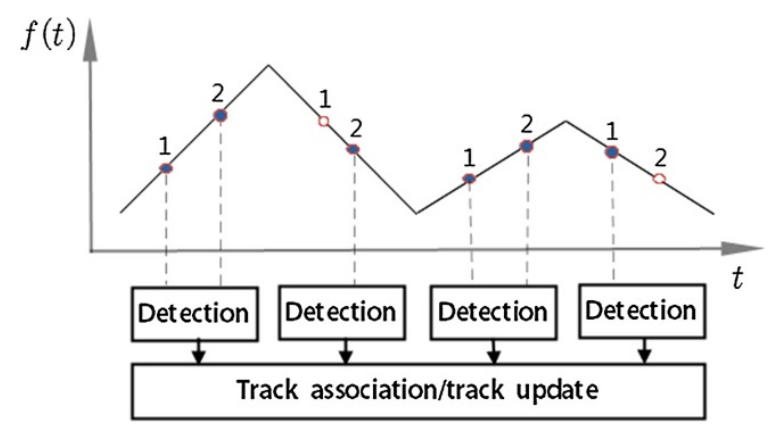

Figure 1 Detections and misses of two targets along a sequence of four chirps of an FMCW radar.

chirp $j$ of radar $m$. Then, the beat frequency $f_{i(j, m), j, m}$ can be represented with [7]

$$
f_{i(j, m), j, m}=\left|a_{j, m} r_{i(j, m), j, m}+b \dot{r}_{i(j, m), j, m}\right|,
$$

where $a_{j, m}=-2 \Delta f_{j, m} /\left(c T_{c}\right), b=-2 / \lambda_{c}$, and $\lambda_{c}$ is the wavelength at the center frequency of the chirp signals, $c$ is the speed of light, and $T_{c}$ is the chirp signal length.

We maintain one track for each target and update it with a sequence of beat frequency measurements that could have originated from the target. The direct use of the beat frequency measurements in track updates is formulated in the sequel. Firstly, we represent the position and velocity of target $t$ in the $x-y$ coordinate frame with the state vector

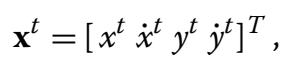

where the superscript $T$ denotes the transpose. The coordinate frame is fixed to a system platform. The discrete white noise acceleration (DWNA) model [11] is employed to characterize the uncertainty in target motion with respect to the platform. The target motion relative to the platform is represented as follows:

$$
\mathbf{x}^{t}(k+1)=\mathbf{F}(k) \mathbf{x}^{t}(k)+\mathbf{v}^{t}(k)
$$

with

$$
\mathbf{F}(k)=\left[\begin{array}{cccc}
1 & T_{k} & 0 & 0 \\
0 & 1 & 0 & 0 \\
0 & 0 & 1 & T_{k} \\
0 & 0 & 0 & 1
\end{array}\right]
$$

where $k$ is a discrete-time index and $T_{k}$ is the time interval between the $k$ th and $(k+1)$ th updates. The vector $\mathbf{v}(k)$ represents a zero-mean white process with covariance matrix

$$
\mathbf{Q}(k)=E\left[\mathbf{v}^{t}(k)\left(\mathbf{v}^{t}(k)\right)^{T}\right]=\left[\begin{array}{cccc}
T_{k}^{4} / 4 & T_{k}^{3} / 2 & 0 & 0 \\
T_{k}^{3} / 2 & T_{k}^{2} & 0 & 0 \\
0 & 0 & T_{k}^{4} / 4 & T_{k}^{3} / 2 \\
0 & 0 & T_{k}^{3} / 2 & T_{k}^{2}
\end{array}\right] \sigma_{v}^{2} .
$$


This model assumes that the target undergoes a constant acceleration during each update period with variance $\sigma_{v}^{2}$, and the accelerations are uncorrelated from period to period.

Let $z_{i(j, m), j, m}(k)$ denote the measurement $i(j, m)$ from radar $m$ and chirp $j$ at $k$. Suppose that the beat frequency measurement originated from target $t$. Then, the measurement is a function of the target state $\mathbf{x}^{t}(k)$, that is,

$$
z_{i(j, m), j, m}(k)=h_{j, m}\left(\mathbf{x}^{t}(k)\right)+w_{i(j, m), j, m}(k),
$$

where $w_{i(j, m), j, m}(k)$ is the white measurement noise at time $k$ with zero mean and variance $R(k)$, and $h_{j, m}\left(\mathbf{x}^{t}(k)\right)$ denotes the true beat frequency given by (1) for the measurement from target $t$. To be more specific,

$$
\begin{aligned}
h_{j, m}\left(\mathbf{x}^{t}(k)\right)= & a_{j, m} r_{j, m}^{t}(k)+b \dot{r}_{j, m}^{t}(k) \\
= & a_{j, m} \sqrt{\left(x^{t}(k)-x_{m}^{s}\right)^{2}+\left(y^{t}(k)-y_{m}^{s}\right)^{2}} \\
& +b \frac{\dot{x}^{t}(k)\left(x^{t}(k)-x_{m}^{s}\right)+\dot{y}^{t}(k)\left(y^{t}(k)-y_{m}^{s}\right)}{\sqrt{\left(x^{t}(k)-x_{m}^{s}\right)^{2}+\left(y^{t}(k)-y_{m}^{s}\right)^{2}}} .
\end{aligned}
$$

Here, $x_{m}^{s}$ and $y_{m}^{s}$ denote the position of radar sensor $m$ in the $x-y$ coordinates fixed to the platform. We also assume the noise sequences $\mathbf{v}^{t}(k)$ and $w_{i(j, m), j, m}(k)$ and the initial state $\mathbf{x}^{t}(0)$ to be mutually independent.

Note that the measurement equation (6) is nonlinear. The estimation of the target states using the beat frequency measurement requires a nonlinear estimator. We use the first-order extended Kalman filter, which is one of the simplest structures for implementing a nonlinear estimator. It relies on a first-order expansion of the nonlinear equation (7) and calculates the state estimate and its covariance matrix recursively [11]. Let $\hat{\mathbf{x}}^{t}(k \mid k)$ denote an approximate conditional mean of the state of target $t$ given the observations up to time $k$, and let $\mathbf{P}^{t}(k \mid k)$ denote its covariance matrix. One cycle of the first-order extended Kalman filter, evolving $\mathbf{x}^{t}(k \mid k)$ and $\mathbf{P}^{t}(k \mid k)$ into $\mathbf{x}^{t}(k+1 \mid k+1)$ and $\mathbf{P}^{t}(k+1 \mid k+1)$, respectively, can be described as follows:

- Time update

Obtain the one-step predicted state of target $t$ and its covariance matrix by evaluating

$$
\begin{aligned}
& \hat{\mathbf{x}}^{t}(k+1 \mid k)=\mathbf{F}(k) \hat{\mathbf{x}}^{t}(k \mid k), \\
& \mathbf{P}^{t}(k+1 \mid k)=\mathbf{F}(k) \mathbf{P}^{t}(k \mid k) \mathbf{F}(k)^{T}+\mathbf{Q}(k) .
\end{aligned}
$$

Obtain the one-step predicted measurement of target $t$ for chirp $j$ of radar $m$ and its variance by

$$
\begin{gathered}
\hat{z}_{j, m}^{t}(k+1 \mid k)=h_{j, m}\left(\hat{\mathbf{x}}^{t}(k+1 \mid k)\right), \\
S_{j, m}^{t}(k+1)=\left(\mathbf{H}_{j, m}^{t}(k+1)\right)^{T} \mathbf{P}^{t}(k+1 \mid k) \mathbf{H}_{j, m}^{t}(k+1)+R(k),
\end{gathered}
$$

where $\mathbf{H}_{j, m}^{t}(k+1)$ is the Jacobian of the scalar $h_{j, m}(\mathbf{x})$ evaluated at $\hat{\mathbf{x}}^{t}(k+1 \mid k)$, that is,

$\mathbf{H}_{j, m}^{t}(k+1)=\frac{1}{\sqrt{\left(x-x_{m}^{s}\right)^{2}+\left(y-y_{m}^{s}\right)^{2}}}$
$\times\left.\left[\begin{array}{c}a_{j, m}\left(x-x_{m}^{s}\right)+b\left\{\dot{x}-\frac{\dot{x}\left(x-x_{m}^{s}\right)^{2}+\dot{y}\left(x-x_{m}^{s}\right)\left(y-y_{m}^{s}\right)}{\left(x-x_{m}^{s}\right)^{2}+\left(y-y_{m}^{s}\right)^{2}}\right\} \\ b\left(x-x_{m}^{s}\right) \\ a_{j, m}\left(y-y_{m}^{s}\right)+b\left\{\dot{y}-\frac{\dot{y}\left(y-y_{m}^{s}\right)^{2}+\dot{x}\left(x-x_{m}^{s}\right)\left(y-y_{m}^{s}\right)}{\left(x-x_{m}^{s}\right)^{2}+\left(y-y_{m}^{s}\right)^{2}}\right\} \\ b\left(y-y_{m}^{s}\right)\end{array}\right]\right|_{\mathbf{x}=\hat{\mathbf{x}}^{t}(k+1 \mid k)} ^{T}$.

- State update

Update the state of target $t$ with new measurement $z_{i(j, m), j, m}(k+1)$ and its covariance matrix by

$$
\begin{aligned}
\hat{\mathbf{x}}^{t}(k+1 \mid k+1)= & \hat{\mathbf{x}}^{t}(k+1 \mid k)+\mathbf{K}_{j, m}^{t}(k+1) \\
& \times\left(z_{i(j, m), j, m}(k+1)-\hat{z}_{j, m}^{t}(k+1 \mid k)\right), \\
\mathbf{P}^{t}(k+1 \mid k+1)= & \mathbf{P}^{t}(k+1 \mid k)-\mathbf{K}_{j, m}^{t}(k+1) S_{j, m}^{t}(k+1) \\
& \times\left(\mathbf{K}_{j, m}^{t}(k+1)\right)^{T},
\end{aligned}
$$

where $\mathbf{K}_{j, m}^{t}(k+1)$ is the Kalman gain:

$$
\mathbf{K}_{j, m}^{t}(k+1)=\mathbf{P}^{t}(k+1 \mid k) \mathbf{H}_{j, m}^{t}(k+1)\left(S_{j, m}^{t}(k+1)\right)^{-1} .
$$

In the above, the state of target $t$ is updated with a new beat frequency measurement $z_{i(j, m), j, m}(k+1)$ from chirp $j$ of radar $m$. Suppose that $M$ radars are employed each with $J$ chirps and suppose that beat frequency measurements from chirp $j$ of radar $m$ are processed to update target states sequentially for $j=1, \ldots, J$ and $m=1, \ldots, M$. The recursion of the state updates for the $M \times J$ chirps will be called as one frame in the sequel. In tracking multiple targets in the presence of clutter, it is required at each update to find the beat frequency measurement $z_{i(j, m), j, m}(k+1)$ that could have originated from target $t$ among possibly numerous measurements from chirp $j$ of radar $m$. That is a measurementto-track association problem addressed in the following section.

\section{Data association and track management}

We maintain one track for each target and update it with beat frequency measurements from an FMCW radar network. In tracking multiple targets in the presence of clutter, however, it is unknown which measurement originated from which track and it is necessary to establish correspondence between tracks and measurements for track updates. The direct track updates in the beat frequency 
measurement space described in Section 2 allow us to update tracks sequentially on a chirp by chirp basis. This sequential processing effectively decomposes the data association problem into a series of 2-D suboptimal assignment problems that can be solved with much less computational effort. The assignment problem is to minimize a cost function, which is the negative log-likelihood ratio $[12,13]$, subject to constraints enforcing assignment rules [10]. In contrast, the measurement-to-track association in the range/range-rate space and in the target state space requires to be carried out for beat frequency measurements from a multiple number of chirps, which becomes a higher-dimensional assignment problem that is computationally much more expensive.

The goal of the 2-D assignment is to find the most likely set of pairs such that each measurement is assigned to one and only one track, or declared not associated, and each track is associated with at most one measurement. Let $N_{j, m}$ denote the number of measurements from chirp $j$ of radar $m$ at time $k+1$, and let $N_{t}$ denote the number of tracks before the state update with the measurements. The 2-D assignment problem is formulated for the set of measurements from chirp $j$ of radar $m$ as follows [9]:

$$
\min _{\rho_{t, i(j, m)}(k+1)} \sum_{t=1}^{N_{t}} \sum_{i(j, m)=0}^{N_{j, m}} c_{t, i(j, m)}(k+1) \rho_{t, i(j, m)}(k+1)
$$

subject to

$$
\begin{aligned}
& \sum_{i(j, m)=0}^{N_{j, m}} \rho_{t, i(j, m)}(k+1)=1, \quad t=1, \ldots, N_{t}, \\
& \sum_{t=0}^{N_{t}} \rho_{t, i(j, m)}(k+1)=1, \quad i(j, m)=1, \ldots, N_{j, m},
\end{aligned}
$$

where $\rho_{t, i(j, m)}(k+1)$ is the binary association variable such that $\rho_{t, i(j, m)}(k+1)=1$ if measurement $z_{i(j, m), j, m}(k+1)$ is associated with track $t$; otherwise, it is set to zero. $c_{t, i(j, m)}(k+1)$ is the negative log-likelihood ratio given by

$$
\begin{aligned}
c_{t, i(j, m)}(k+1)= & \frac{1}{2}\left[z_{i(j, m), j, m}(k+1)-\hat{z}_{j, m}^{t}(k+1 \mid k)\right]^{T} . \\
& S_{j, m}^{t}(k+1)^{-1}\left[z_{i(j, m), j, m}(k+1)-\hat{z}_{j, m}^{t}(k+1 \mid k)\right] \\
& +\ln \frac{\lambda\left|2 \pi S_{j, m}^{t}(k+1)\right|^{1 / 2}}{P_{D}^{t}}
\end{aligned}
$$

if measurement $z_{i(j, m), j, m}(k+1)$ is assigned to track $t$, and

$$
c_{t, 0}(k+1)=-\ln \left[1-P_{D}^{t}\right],
$$

if no measurement is assigned to track $t$. Note that the measurement prediction error and its residual variance are used in (19) to evaluate the cost function $c_{t, i(j, m)}(k+1)$.
The parameter $\lambda$ denotes the spatial density of the clutter and new targets, which is a design parameter. $P_{D}^{t}$ is the detection probability of target $t$. The 2-D assignment problem is a constrained integer programming problem. A variety of both simple and complex methods have been developed to solve the problem, including the Munkres algorithm, Auction algorithm, or JVC algorithm $[9,14]$. We can utilize any of these algorithms to solve the 2-D assignment problem. In our experiments, we used the Munkres algorithm. A goodness-of-fit test can also be employed in high target density situations to further reduce the computational load by partitioning the 2-D assignment problem into smaller subproblems [9]. The test screens out unlikely candidate assignments to build disjoint candidate assignments and put them into separate assignment subproblems. The negative log-likelihood ratio (19) is utilized as a metric for the screening.

The solution of the 2-D assignment problem yields the most likely set of measurement-to-track pairs which establishes correspondence between tracks and beat frequency measurements. The measurement of each pair is used to update its associated track. Those measurements that are not associated with any tracks are processed to initiate new tracks. The new initiated tracks are classified to be candidate tracks. Some of the candidate tracks are promoted to become established tracks depending on track quality, and others are deleted. Figure 2 presents a flow diagram of our track management system. When a new set of beat frequency measurements from a chirp is forwarded to the track management system, they are used to update established tracks in the first phase. Measurements that have updated established tracks are removed from the set, and the remaining residual measurements are forwarded to update candidate tracks in the second phase. The measurements that have updated candidate tracks are removed from the measurement set, and the remaining measurements are used to initialize new candidate tracks.

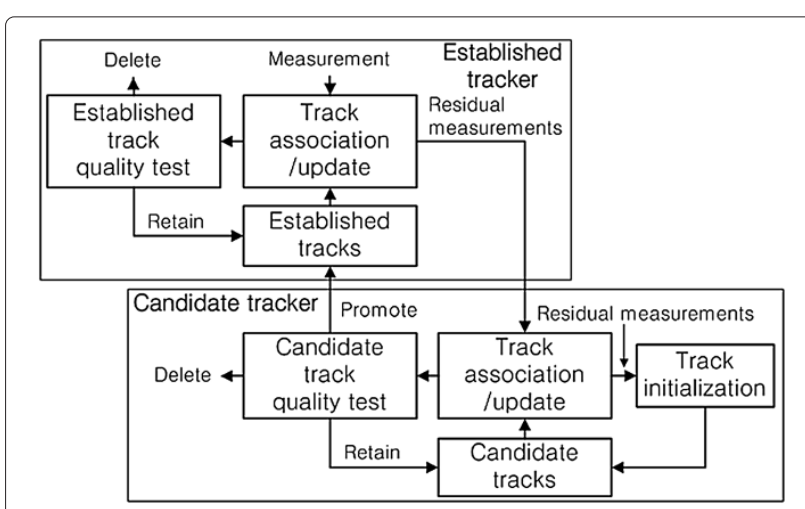

Figure 2 Flow diagram of track management system. 


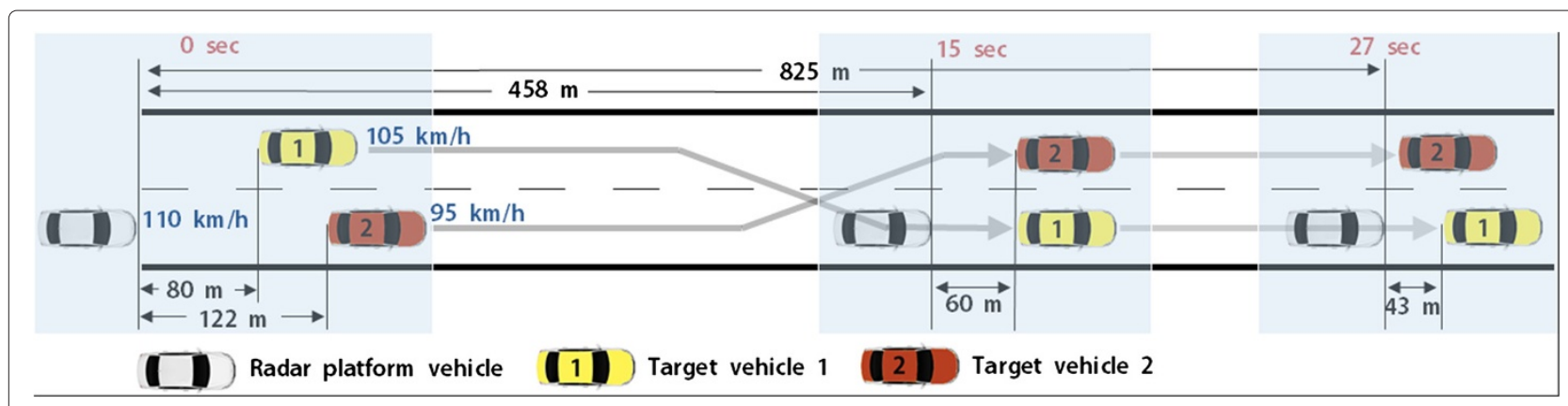

Figure 3 The geometry of scenario I of tracking two target vehicles.

The quality of established and candidate tracks is tested with an $M$-out-of- $N$ logic whether or not the tracks are well maintained. The $M$-out-of- $N$ logic tests the track quality based on the number of successful track updates in the last $N$ attempts [15]. The tracks with poor update records are deleted from the established and candidate tracks. When a candidate track meets a certain quality level, it is promoted to be an established track. In this structure, established tracks can prevent existing candidate tracks to be updated and new candidate tracks to be formed on new targets. In the meantime, this reduces the chance that candidate tracks interfere with established tracks to degrade.

\section{Numerical experiments}

In our numerical experiments, the FMCW radar network consists of four radar sensors collocated at a platform. The platform coordinate system has the origin at the center of the platform, and the $y$-axis of the coordinates directs

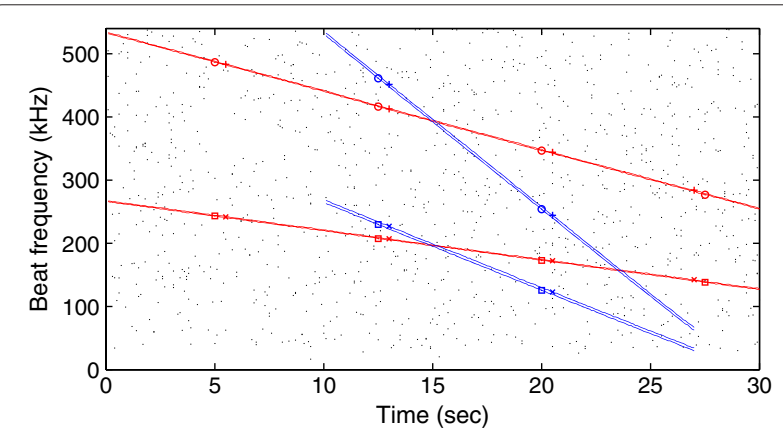

Figure 4 The beat frequency trajectories of the two targets in scenario I. The trajectories are obtained from four chirps of radar sensor 1. The dots indicate the beat frequency measurements from clutter for parameter $\lambda_{f}=1$. Legend: red line with circle markers: target 1, chirp 1 (up); red line with + markers: target 1, chirp 2 (down); red line with square markers: target 1, chirp 3 (up); red line with $x$ markers: target 1, chirp 4 (down); blue line with circle markers: target 2, chirp 1 (up); blue line with + markers: target 2, chirp 2 (down); blue line with square markers: target 2, chirp 3 (up); blue line with $x$ markers: target 2, chirp 4 (down); black dots: clutter. towards the front. The four radar sensors are placed at $-75,-25,25$, and $75 \mathrm{~cm}$ along the $x$-axis of the coordinates. The detection range is assumed to be $80 \mathrm{~m}$, and the field of view in azimuth is $60^{\circ}$. The center frequency is $77 \mathrm{GHz}$. Each radar transmits two pairs of up/down chirps, each with a chirp signal length of $1 \mathrm{~ms}$, and the sweep frequencies of the first and second pairs are 1 and $0.5 \mathrm{GHz}$, respectively. The beat frequency measurements are obtained with 16 chirps ( 4 chirps per radar) per frame and sequentially processed at every $6.25 \mathrm{~ms}$. The frame rate is $10 \mathrm{~Hz}$. The standard deviation of the beat frequency measurement error is $400 \mathrm{~Hz}$. The numerical experiments are performed to evaluate the performance of the proposed track management system in maintaining vehicle tracks. The experiments, however, are not intended to address automotive applications, where radar signals are coherently processed [3,4].

Monte Carlo simulations were performed to evaluate tracking performances for two scenarios. Scenario I is illustrated in Figure 3, which depicts the geometrical scenario of two target vehicles and the radar platform vehicle. In the scenario, target 1 was tracked over $[0 \mathrm{~s}, 30 \mathrm{~s}]$, and target 2 was tracked over $[10 \mathrm{~s}, 27 \mathrm{~s}]$ where each individual target lies in the field of view. Targets 1 and 2 change lanes over time intervals $[10 \mathrm{~s}, 13 \mathrm{~s}$ ] and [12 s, $15 \mathrm{~s}$ ], respectively, while maintaining a constant speed. The lane width is $4 \mathrm{~m}$. The simulations were performed for nonunity detection

Table 1 Number of simulation runs in terms of time required to establish track 1 (scenario I)

\begin{tabular}{cccccccc}
\hline & \multicolumn{8}{c}{ Time required in seconds to establish track 1 } \\
$\boldsymbol{P}_{\boldsymbol{D}}$ & $\boldsymbol{\lambda}_{\boldsymbol{f}}$ & $\mathbf{0 . 1}$ & $\mathbf{0 . 2}$ & $\mathbf{0 . 3}$ & $\mathbf{0 . 4}$ & $\mathbf{0 . 5}$ & Average \\
\hline 0.7 & 0.33 & 11 & 977 & 11 & - & 1 & 0.20 \\
& 1.0 & 116 & 813 & 64 & 5 & 2 & 0.20 \\
0.8 & 0.33 & 61 & 931 & 8 & - & - & 0.19 \\
& 1.0 & 252 & 724 & 23 & 1 & - & 0.18 \\
0.9 & 0.33 & 298 & 699 & 3 & - & - & 0.17 \\
& 1.0 & 520 & 475 & 5 & - & - & 0.15 \\
\hline
\end{tabular}


Table 2 Number of track losses of target 1 (scenario I)

\begin{tabular}{ccccc}
\hline \multicolumn{3}{c}{$\mathbf{0 . 2 \mathrm { s }}$} & \multicolumn{2}{c}{$\mathbf{0 . 5 \mathrm { s }}$} \\
$P_{D}$ & $\lambda_{f}=\mathbf{0 . 3 3}$ & $\lambda_{f}=\mathbf{1 . 0}$ & $\lambda_{f}=\mathbf{0 . 3 3}$ & $\lambda_{f}=\mathbf{1 . 0}$ \\
\hline 0.7 & - & 11 & - & 1 \\
0.8 & - & 6 & - & 1 \\
0.9 & - & 1 & - & - \\
\hline
\end{tabular}

Given that the track has been maintained as established at 0.2 and $0.5 \mathrm{~s}$.

probability in the presence of clutter. The beat frequency from clutter is assumed to be uniformly distributed over the beat frequency range corresponding to the detection range with null range-rate. The number of clutter has a Poisson distribution with parameter $\lambda_{f}$. Tracking performances are insensitive to parameter $\lambda$ in (19), and we set it to the inverse value of the beat frequency range. Figure 4 shows the beat frequency trajectories of the two targets from four chirps of radar sensor 1 under the scenario. Target 1 stays in the field of view over the 30-s interval, and we can see that target 2 pops up at $10 \mathrm{~s}$ and continues until $27 \mathrm{~s}$. The figure also shows that the beat frequencies from the targets cross each other at approximately $15 \mathrm{~s}$, where the targets are close in range as can be seen in Figure 3 . The dots in the figure indicate the beat frequency measurements from clutter for parameter $\lambda_{f}=1$.

One-point initialization was utilized to initialize the state vector and its covariance matrix of a target indexed $t$ with beat frequency measurement $z_{i(j, m), j, m}(0): \hat{\mathbf{x}}^{t}(0 \mid 0)=\left[\hat{x}^{t}(0 \mid 0) \hat{\dot{x}}^{t}(0 \mid 0) \hat{y}^{t}(0 \mid 0) \hat{\dot{y}}^{t}(0 \mid 0)\right]^{T}=$ $\left[\begin{array}{lll}0 & 0 & \left|z_{i(j, m), j, m}(0) / a_{j, m}\right|-10\end{array}\right]^{T}$ and $\mathbf{P}^{t}(0 \mid 0)=\operatorname{diag}\{10,10$, $10,100\}$. The parameter $\sigma_{v}$ in (5) was set to $10 \mathrm{~m} / \mathrm{s}^{2}$. The $M$-out-of- $N$ logics were implemented to assess the track quality of candidate and established tracks. A candidate track is promoted to an established track with a 9-out-of-16 logic, it is deleted with a 6-out-of-16 logic, and it is retained as a candidate track, otherwise. An established track is decided to retain or delete based on a 12-out-of-32 logic.

A simulation of 1,000 Monte Carlo runs was performed for $P_{D}=0.7,0.8,0.9$ and $\lambda_{f}=0.33,1.0$. Firstly, we

Table 3 Number of simulation runs in terms of time required to establish track 2 (scenario I)

\begin{tabular}{cccccccc}
\hline & \multicolumn{8}{c}{ Time required in seconds to establish track 2 } \\
$\boldsymbol{P}_{\boldsymbol{D}}$ & $\boldsymbol{\lambda}_{\boldsymbol{f}}$ & $\mathbf{0 . 1}$ & $\mathbf{0 . 2}$ & $\mathbf{0 . 3}$ & $\mathbf{0 . 4}$ & $\mathbf{0 . 5}$ & Average \\
\hline 0.7 & 0.33 & 154 & 794 & 51 & 1 & - & 0.19 \\
& 1.0 & 196 & 721 & 65 & 14 & 4 & 0.19 \\
0.8 & 0.33 & 243 & 739 & 17 & 1 & - & 0.18 \\
& 1.0 & 300 & 668 & 30 & 2 & - & 0.17 \\
0.9 & 0.33 & 397 & 596 & 7 & - & - & 0.16 \\
& 1.0 & 446 & 538 & 15 & 1 & - & 0.16 \\
\hline
\end{tabular}

Table 4 Number of track losses of target 2 (scenario I)

\begin{tabular}{ccccc}
\hline & \multicolumn{2}{c}{$\mathbf{0 . 2 \mathrm { s }}$} & \multicolumn{2}{c}{$\mathbf{0 . 5 \mathrm { s }}$} \\
$\boldsymbol{P}_{\boldsymbol{D}}$ & $\lambda_{\boldsymbol{f}}=\mathbf{0 . 3 3}$ & $\lambda_{\boldsymbol{f}}=\mathbf{1 . 0}$ & $\lambda_{\boldsymbol{f}}=\mathbf{0 . 3 3}$ & $\boldsymbol{\lambda}_{\boldsymbol{f}}=\mathbf{1 . 0}$ \\
\hline 0.7 & 4 & 26 & 1 & 11 \\
0.8 & 1 & 8 & - & 3 \\
0.9 & - & 2 & - & - \\
\hline
\end{tabular}

Given that the track has been maintained as established at 0.2 and $0.5 \mathrm{~s}$.

obtained the number of frames required to establish a track following the frame with the first target detection. Table 1 lists the number of simulation runs in terms of the frame time required to establish track 1 . In our simulations, the frame time corresponds to $0.1 \mathrm{~s} \times$ (number of frames). The track was established very fast in $0.5 \mathrm{~s}$ in all the simulation runs. The minimum average time was $0.15 \mathrm{~s}$ when $P_{D}=0.9$ and $\lambda_{f}=1$, and the maximum was $0.20 \mathrm{~s}$ when $P_{D}=0.7$ and $\lambda_{f}=0.33$. The table shows that a higher probability of detection reduces the time required to establish a track and, likewise, a higher density of clutter also reduced the time. Note that the clutter density is proportional to the parameter $\lambda_{f}$. Beat frequency measurements from clutter can lie near the beat frequency corresponding to an undetected target of a candidate track. In this case, they can help the candidate track to pass more easily the promotion quality test that is based on the 9-out-of-16 logic. On the contrary, the clutter measurements can also interfere and destabilize a candidate track to defer the promotion. The occurrence of the deferred promotion can be seen in the table. In the simulation runs, a track is declared lost if the track has been established but fails to maintain its established status. Table 2 lists the number of track losses of target 1 , given that the track has been maintained as established at 0.2 and $0.5 \mathrm{~s}$, respectively, among 1,000 simulation runs. It indicates that the probability of track loss is very low and that the track management system can maintain tracks in a reliable manner.

Table 3 lists the number of simulation runs in terms of the time required to establish track 2 . The track was also established very fast within a half second in all the simulation runs, and the average times required to establish track 2 are comparable with those for the case of track 1 . The clutter interference to candidate tracks is also obvious for track 2 . When the clutter density is higher, the number of simulation runs in Table 3 increased significantly for

Table 5 Number of established false tracks (scenario I)

\begin{tabular}{ccc}
\hline $\boldsymbol{P}_{\boldsymbol{D}}$ & $\boldsymbol{\lambda}_{\boldsymbol{f}} \mathbf{=} \mathbf{0 . 3 3}$ & $\boldsymbol{\lambda}_{\boldsymbol{f}}=\mathbf{1 . 0}$ \\
\hline 0.7 & 38 & 30 \\
0.8 & 11 & 50 \\
0.9 & 8 & 47 \\
\hline
\end{tabular}




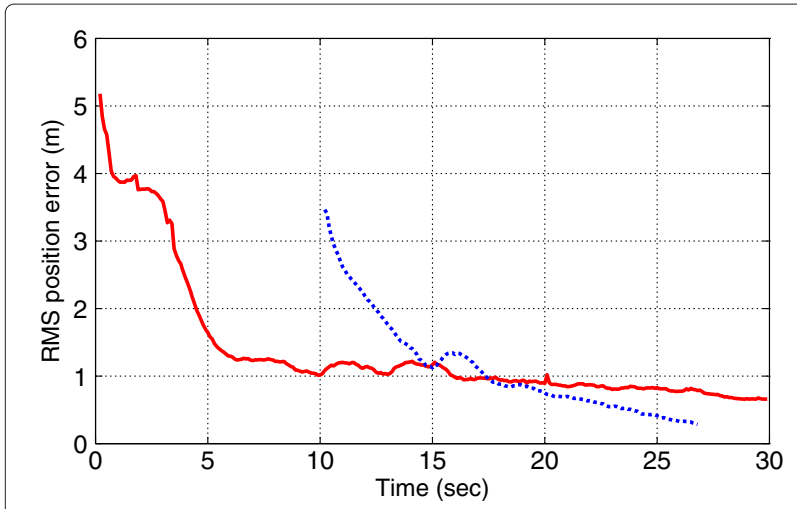

Figure 5 RMS error of position estimates for $P_{D}=0.7$ and $\lambda_{f}=1$ (scenario I). Legend: red solid line: track 1 , blue dotted line: track 2.

$0.3 \mathrm{~s}$ or greater. Note that our simulation starts at $0 \mathrm{~s}$, and track 1 also starts at the same instant under the circumstances that no spurious tentative tracks exist. In contrast, track 2 is initiated at $10 \mathrm{~s}$ where there is a higher possibility that there already exist spurious candidate tracks that lie near the target position but originated from clutter. The possibility is stronger as the clutter density increases. The spurious tracks and beat frequency measurements that originated from clutter can interfere to reduce the time required in establishing a new track in some cases, but they can also interfere to defer a candidate track to promote to the established status in other cases. The spurious tracks can destabilize established tracks and lead to track losses. As a consequence, track 2 is in an unfavorable condition compared to track 1 in establishing its track. The number of track losses slightly increased for track 2 among the 1,000 simulation runs (see Table 4). Clutter measurements can establish false tracks, and the number of established false tracks tends to increase as the clutter density increases (see Table 5). When the number of false tracks is too large to manage, we can implement a more

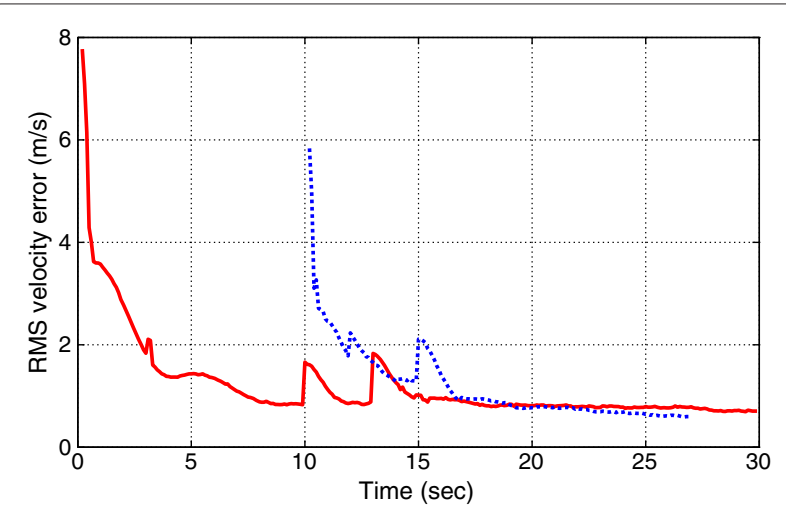

Figure 6 RMS error of velocity estimates for $P_{D}=0.7$ and $\lambda_{f}=1$ (scenario I). Legend: red solid line: track 1, blue dotted line: track 2.

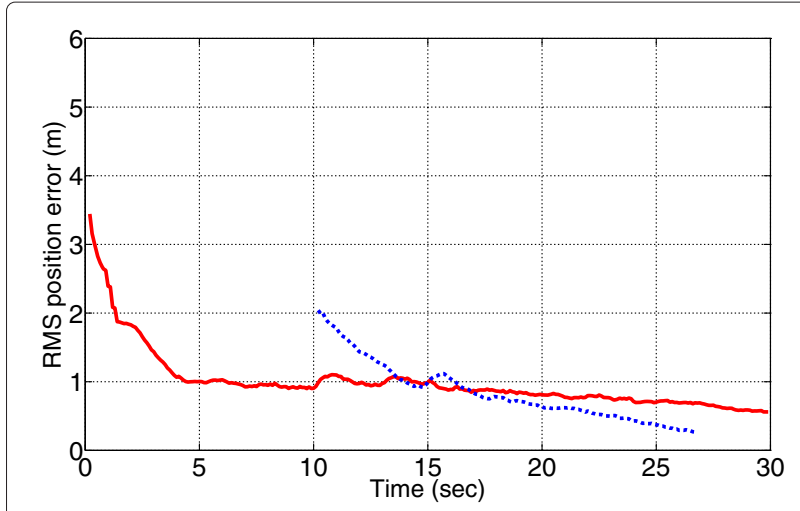

Figure 7 RMS error of position estimates for $P_{D}=0.9$ and $\lambda_{f}=\mathbf{0 . 3 3}$ (scenario I). Legend: red solid line: track 1, blue dotted line: track 2 .

strict promotion quality test. This, however, can increase the time required in establishing a track.

The root-mean-squared error (RMSE) of target position and velocity estimates were obtained for tracks that were not lost. The results are presented starting from $0.2 \mathrm{~s}$ after track initialization in Figures 5 and 6 for $P_{D}=0.7$ and $\lambda_{f}=1$ and in Figures 7 and 8 for $P_{D}=0.9$ and $\lambda_{f}=0.33$. The former and the latter correspond to the least and the most favorable conditions, respectively, in the simulations. Figures 5 and 6 show that the estimation errors decrease fast as the time increases and become less than 4 and $5 \mathrm{~m} / \mathrm{s}$ in $1 \mathrm{~s}$ after the first detection. Figure 5 shows that the position error of track 2 decreases to become less than that of track 1 . This can be explained by the fact that the target observability of the collocated FMCW radars improves as the targets get closer to the platform [11]. Note that the observability of target 2 improves faster since it approaches faster and closer. It was also observed that cross-range error is the dominant component of the position error in the experiments. Figure 6 shows that

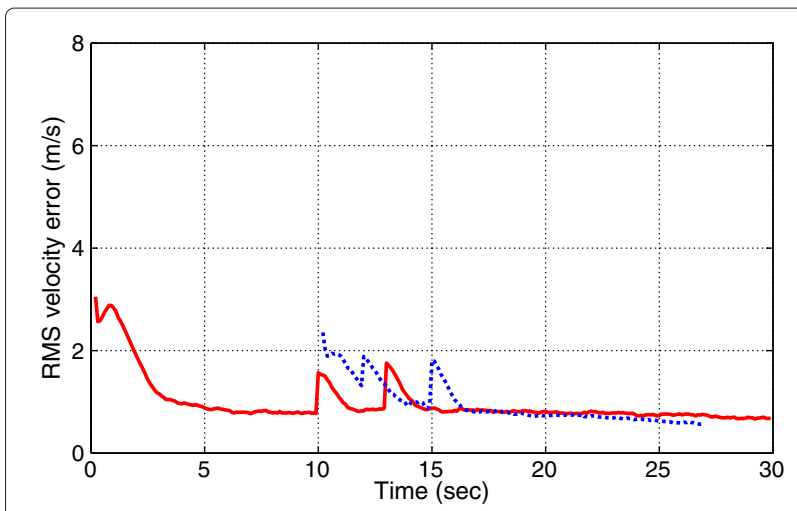

Figure 8 RMS error of velocity estimates for $P_{D}=0.9$ and $\lambda_{f}=\mathbf{0 . 3 3}$ (scenario I). Legend: red solid line: track 1, blue dotted line: track 2 . 
Table 6 Number of simulation runs for $P_{D}=0.9$ and $\lambda_{f}=0.33$ (scenario II)

\begin{tabular}{lccccccc}
\hline & \multicolumn{7}{c}{ Time required in seconds to establish a track } \\
& $\mathbf{0 . 1}$ & $\mathbf{0 . 2}$ & $\mathbf{0 . 3}$ & $\mathbf{0 . 4}$ & $\mathbf{0 . 5}$ & $\mathbf{0 . 6}$ & Average \\
\hline Track 1 & 282 & 716 & 1 & 1 & - & - & 0.17 \\
Track 2 & 422 & 567 & 11 & - & - & - & 0.16 \\
Track 3 & 435 & 556 & 7 & 2 & - & - & 0.16 \\
Object 1 & 351 & 558 & 71 & 16 & 3 & 1 & 0.18 \\
Object 2 & 359 & 553 & 69 & 16 & 3 & - & 0.18 \\
Object 3 & 371 & 540 & 74 & 15 & - & - & 0.17 \\
Object 4 & 332 & 577 & 78 & 10 & 1 & 2 & 0.18 \\
Object 5 & 360 & 550 & 71 & 15 & 4 & - & 0.18 \\
Object 6 & 352 & 551 & 81 & 16 & - & - & 0.18 \\
Object 7 & 349 & 559 & 74 & 16 & 1 & - & 0.18 \\
Object 8 & 363 & 559 & 60 & 13 & 3 & 2 & 0.17 \\
\hline
\end{tabular}

In terms of time required to establish a track.

sharp increases in the velocity error occur at 10 and $13 \mathrm{~s}$ for target 1 and at 12 and $15 \mathrm{~s}$ for target 2 . Note that the time instants are the moments at which the targets start and finish to change their lanes. The lane changes result in the abrupt changes in target velocity that cause the sharp increases in the velocity error. Similar arguments hold for the results presented in Figures 7 and 8 for $P_{D}=0.9$ and $\lambda_{f}=0.33$. As expected, the estimation accuracy improved since this tracking condition is much more favorable.

The same Monte Carlo simulation was performed for scenario II which includes, in addition to the geometry of scenario I presented in Figure 3, a third lane and one target vehicle (target 3 ) on the lane. The target is positioned at $82 \mathrm{~m}$ ahead of the platform and stays in the lane with a speed of $105 \mathrm{~km} / \mathrm{h}$. Stationary objects are also placed with $100 \mathrm{~m}$ apart along the left shoulder of the

Table 7 Number of track losses of each target for $P_{D}=0.9$ and $\lambda_{f}=0.33$ (scenario II)

\begin{tabular}{lcc}
\hline & $\mathbf{0 . 2}$ & $\mathbf{0 . 5}$ \\
\hline Track 1 & - & - \\
Track 2 & - & - \\
Track 3 & - & - \\
Object 1 & 19 & 2 \\
Object 2 & 29 & 7 \\
Object 3 & 23 & 4 \\
Object 4 & 24 & 9 \\
Object 5 & 20 & 8 \\
Object 6 & 17 & 7 \\
Object 7 & 31 & 7 \\
Object 8 & 28 & 11 \\
\hline
\end{tabular}

Given that its track has been maintained as established at 0.2 and $0.5 \mathrm{~s}$.

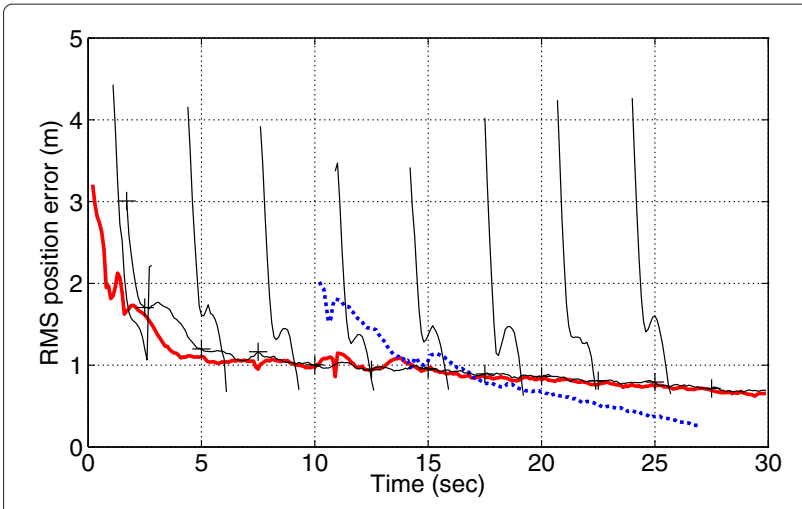

Figure 9 RMS error of position estimates for $P_{D}=0.9$ and $\lambda_{f}=\mathbf{0 . 3 3}$ (scenario II). Legend: red solid line: track 1, blue dotted line: track 2, black thin solid line with + markers: track 3 , thin black solid lines: objects.

highway ( $-7 \mathrm{~m}$ in the $x$ coordinate of the platform). In the scenario, the platform passes eight stationary objects over the 30-s interval, and each object lies in the field of view for approximately $2.3 \mathrm{~s}$. The simulation results for $P_{D}=0.9$ and $\lambda_{f}=0.33$ are presented in Tables 6 and 7 and Figures 9 and 10. The results for target tracks are consistent with those of scenario I. The tracks of stationary objects were established in $0.6 \mathrm{~s}$ in all the simulation runs (see Table 6). The table also shows that $0.3 \mathrm{~s}$ or greater time was required in more simulation runs to establish a track for a stationary object, compared to a target track. The lag is due to the fact that the state vector of the extended Kalman filter was unfavorably initialized for the stationary objects and the objects are approaching much faster than the targets. The objects were placed along the left shoulder, and their initial lateral position error is much larger compared to those of targets in a highway lane. These adverse conditions can disrupt candidate

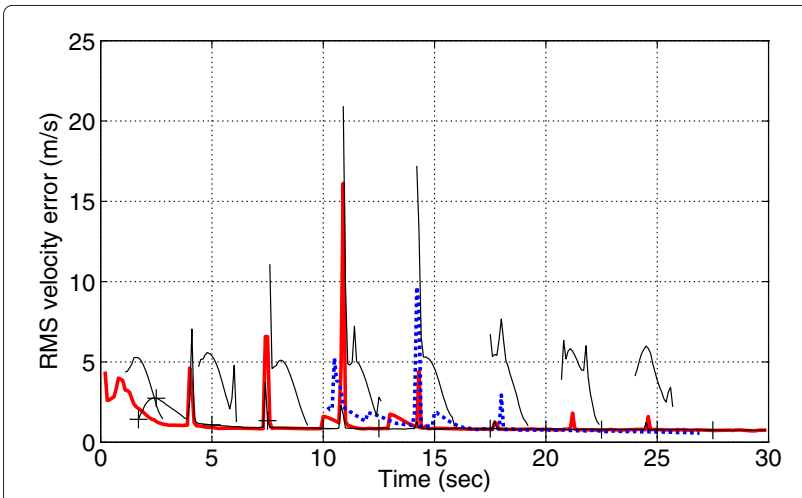

Figure 10 RMS error of velocity estimates for $P_{D}=0.9$ and $\lambda_{f}=\mathbf{0 . 3 3}$ (scenario II). Legend: red solid line: track 1, blue dotted line: track 2, black thin solid line with + markers: track 3 , thin black solid lines: objects. 
tracks to defer their promotion. The unfavorable conditions also affect track maintenance in the early stage of tracking. Table 7 shows that track loss of stationary objects can occur with probability of $1 \%$, given that their tracks have been maintained as established at $0.5 \mathrm{~s}$. The RMS estimate errors of the stationary objects are presented in Figures 9 and 10 starting from $0.4 \mathrm{~s}$ after track initialization. Due to the unfavorable initialization, the estimates start with a large position error. However, the position errors decrease fast and become close to the corresponding estimate errors of tracks 1 and 3 (see Figure 9). In Figure 10, we can observe multiple spikes in the velocity error. The spikes are owing to the interferences between targets and objects, and the interferences occur when the objects are in the initial stage of tracking. It can be seen from the figure that target 1 interferes with objects $2,3,4$, and 5 , and target 2 interferes with objects 4,5 , and 6 . Note that the targets interfere with a stationary object when they stay in the first lane, which is close to the left shoulder and the object.

\section{Conclusions}

In this paper, we formulated a multiple-target tracking problem for an FMCW radar network in the presence of clutter and presented a track management system for solving it. The track management system receives beat frequency measurements from the FMCW radar network and associates them to tracks directly in the beat frequency measurement space. The direct association eliminates range/range-rate calculations and multilateration processing, and it allows to process beat frequency measurements sequentially on a chirp by chirp basis. The sequential processing effectively decomposes the measurement-to-track association problem into a series of two-dimensional assignment problems that can be solved with much less computational effort. The solution to the measurement-to-track association is utilized to initiate and form new tracks and to update or delete existing tracks. Monte Carlo simulations were performed to evaluate the track management system. The simulation results indicate that the track management system maintains tracks with a very low probability of track loss and provides accurate estimates of target position and velocity.
Engineering, Kyungpook National University, 1370 Sankyuk-dong, Pook-gu, Daegu 702-701, South Korea.

Received: 7 March 2013 Accepted: 4 October 2013

Published: 17 October 2013

\section{References}

1. AG Stove, Linear FMCW radar techniques. IEE Proceedings-F Radar, Sonar Navigation. 139(5), 343-350 (1992)

2. H Rohling, in 11th International Radar Symposium 2010. Milestones in radar and the success story of automotive radar systems (Vilnius, 16-18 June 2010), pp. 1-6

3. J Massen, M Frei, W Menzel, U Möller, A $79 \mathrm{GHz}$ SiGe short-range radar sensor for automotive applications. Int. J. Microw. Wireless Technol. 5(1), 5-14 (2013)

4. H Rohling, M Kronauge, in Waveform Design and Diversity for Advanced Radar Systems, ed. by F Gini, A De Maio, and Patton L. Continuous waveforms for automotive radar systems (IET, London, 2012), pp. 173-205

5. A Dzvonkovskaya, K-W Gurgel, H Rohling, T Schlick, HF radar WERA application for ship detection and tracking. Eur. J. Navigation. 7(3), 18-25 (2009)

6. S Clark, L Perletta, in 19th ITS World Congress. ClearWay radar for the detection and enforcement of illegal vehicle lane change and tail-gating events (Vienna, 22-26 Oct 2012)

7. H Rohling, in Advances in Sensing with Security Applications, NATO Security Through Science Series. Some radar topics: waveform design, range CFAR and target recognition, vol. 2 (Springer, Dordrecht, 2006), p. 293

8. D Vivet, P Checchin, R Chapuis, P Faure, R Rouveure, MO Monod, A mobile ground-based radar sensor for detection and tracking of moving objects. EURASIP J. Adv. Signal Process (2012). doi:10.1186/1687-6180-2012-45

9. Y Bar-Shalom, W Blair (eds.), Multitarget-Multisensor Tracking:Applications and Advances, vol. 3 (Artech House, Norwood, 2000)

10. S Deb, M Yeddanapudi, K Pattipati, Y Bar-Shalom, A generalized S-D assignment algorithm for multisensor-multitarget state estimation. IEEE Trans. Aerosp. Electron. Syst. 33(2), 523-538 (1997)

11. Y Bar-Shalom, $X \mathrm{Li}$, T Kirubarajan, Estimation with Applications to Tracking and Navigation (Wiley, New York, 2001)

12. Y Bar-Shalom, SS Blackman, RJ Fitzgerald, Dimensionless score function for multiple hypothesis tracking. IEEE Trans. Aerosp. Electron. Syst. 43(1), 392-400 (2007)

13. LM Kaplan, Y Bar-Shalom, W Blair, Assignment costs for multiple sensor track-to-track association. IEEE Trans. Aerosp. Electron. Syst. 44(2), 655-677 (2008)

14. KR Pattipatti, S Deb, Y Bar-Shalom, RB Washburn, A new relaxation algorithm and passive sensor data association. IEEE Trans. Automatic Control. 37(2), 198-213 (1992)

15. SS Blackman, Multiple-Target Tracking with Radar Applications (Artech House, Norwood, 1986)

doi:10.1186/1687-6180-2013-159

Cite this article as: Kim and Hong: Multiple-target tracking and track management for an FMCW radar network. EURASIP Journal on Advances in Signal Processing 2013 2013:159.

\section{Competing interests}

The authors declare that they have no competing interests.

\section{Acknowledgements}

This work was supported by the IT R\&D program of MSIP/KEIT (10041145, Self-Organizing Software Platform for Welfare Devices), by the Basic Science Research Program through the NRF funded by the Ministry of Education (grant no. 2011-0013475), and by Kyungpook National University Research Fund, 2012.

\section{Author details}

${ }^{1}$ Samsung Techwin R\&D Center, 701 Sampyeong-dong, Bundang-gu, Seongnam-si, Gyeonggi-do 463-400, South Korea. ${ }^{2}$ School of Electronics 